\title{
BIFURCATION ANALYSIS OF A DELAYED EPIDEMIC MODEL WITH DIFFUSION
}

\author{
Changuin Xu and MaOxin Liao
}

\begin{abstract}
In this paper, a class of delayed epidemic model with diffusion is investigated. By analyzing the associated characteristic transcendental equation, its linear stability is investigated and Hopf bifurcation is demonstrated. Some explicit formulae determining the stability and the direction of the Hopf bifurcation periodic solutions bifurcating from Hopf bifurcations are obtained by using the normal form theory and center manifold theory. Some numerical simulation are also carried out to support our analytical findings. Finally, biological explanations and main conclusions are given.
\end{abstract}

\section{Introduction}

In recent years, there has been a growing interest in the study of the dynamical behavior (including stable, unstable, persistent and oscillatory behavior) of epidemic models which plays an important role in the study of mathematical epidemiology. Great attention has been paid to the dynamics properties of the epidemic models which have significant biological background. Many excellent and interesting results have been obtained [1-5, 7, 9-11, 13-14, 16-21, 25-26]. In 1997, Zhang and Fang [24] investigated dispersal properties of the following epidemic model:

$$
\left\{\begin{array}{l}
\dot{s}_{1}=-k_{1} s_{1} I_{1}+s_{1}\left(a_{1}-s_{1}\right)+\varepsilon\left(s_{2}-s_{1}\right), \\
\dot{s}_{2}=s_{2}\left(a_{2}-s_{2}\right)+\varepsilon\left(s_{1}-s_{2}\right), \\
\dot{I}_{1}=k_{1} s_{1} I_{1}-b I_{1}-c I_{1},
\end{array}\right.
$$

where $s_{1}, s_{2}$ represent the susceptible numbers in place 1 at time $t$ and the susceptible numbers in place 2 at time $t$, respectively, $I_{1}$ denotes the infective numbers in place 1 at time $t, k$ is infective coefficient, $k s_{1}$ is infective rate, $a_{1}, a_{2}$ are the carrying capacities in place 1 and in place 2 , respectively, $b$ is

Received November 14, 2009.

2010 Mathematics Subject Classification. 34K20, 34C25.

Key words and phrases. epidemic model, diffusion, delay, stability, Hopf bifurcation.

This work is supported by National Natural Science Foundation of China (No.10771215 and No.10771094), the Scientific Research Fund of Hunan Provincial Education Department (10C0560) and the Scientific Research Initializing Foundation of Hunan Institute of Engineering (No.0744). 
death rate of infective numbers, $c$ is eliminative rate of the infective numbers, $c$ is diffusion rate between the susceptible numbers. All the parameters are positive constants.

Considering the factor that the infective numbers in place 1 will stay latent before becoming infectious, we think that time delay has important biologic meaning in epidemic models and so it is more plausible to incorporate the time delay into the model. Based on the point of view, we revise model (1) into the following delayed epidemic model with diffusion:

$$
\left\{\begin{array}{l}
\dot{s}_{1}=-k_{1} s_{1} I_{1}(t-\tau)+s_{1}\left(a_{1}-s_{1}\right)+\varepsilon\left(s_{2}-s_{1}\right), \\
\dot{s}_{2}=s_{2}\left(a_{2}-s_{2}\right)+\varepsilon\left(s_{1}-s_{2}\right) \\
\dot{I}_{1}=k_{1} s_{1} I_{1}(t-\tau)-b I_{1}(t-\tau)-c I_{1}(t-\tau)
\end{array}\right.
$$

where all the parameters are positive constants. The more detail biological meaning of the coefficients of the system (2) are same as those in (1) and one can see [24].

In this paper, we study the stability, the local Hopf bifurcation for system (2). To the best of our knowledge, it is the first time to deal with the research of Hopf bifurcation for the model (2).

The remainder of the paper is organized as follows. In Section 2, we investigate the stability of the boundary equilibrium and the occurrence of local Hopf bifurcations. In Section 3, the direction and stability of the local Hopf bifurcation are established. In Section 4, numerical simulations are carried out to illustrate the validity of the main results. Biological explanations and some main conclusions are drawn in Section 5.

\section{Stability of the boundary equilibrium and local Hopf bifurcations}

In this section, we shall study the stability of the boundary equilibrium and the existence of local Hopf bifurcations. Throughout the paper, we assume that (H1). The equation

$$
\left\{\begin{array}{l}
s_{1}\left(a_{1}-s_{1}\right)+\varepsilon\left(s_{2}-s_{1}\right)=0 \\
s_{2}\left(a_{2}-s_{2}\right)+\varepsilon\left(s_{1}-s_{2}\right)=0
\end{array}\right.
$$

has an unique positive root.

It is easy to see that if the condition (H1) holds, then Eq.(2) has an unique boundary equilibrium $E_{0}\left(s_{1}^{*}, s_{2}^{*}, I_{1}^{*}\right)$, where $I_{1}^{*}=0$ and $s_{1}^{*}, s_{2}^{*}$ are the positive roots of Eq.(3).

Let $\bar{s}_{1}(t)=s_{1}(t)-s_{1}^{*}, \bar{s}_{2}(t)=s_{2}(t)-s_{2}^{*}, \bar{I}_{1}(t)=I_{1}(t)$ and still denote $\bar{s}_{i}(t)(i=1,2), \bar{I}_{1}(t)$ by $s_{i}(t)(i=1,2), I_{1}(t)$, respectively. Then $(2)$ becomes

(4) $\left\{\begin{array}{l}\dot{s}_{1}(t)=\left(a_{1}-2 s_{1}^{*}-\varepsilon\right) s_{1}+\varepsilon s_{2}-k s_{1}^{*} I_{1}(t-\tau)-s_{1}^{2}-k s_{1} I_{1}(t-\tau), \\ \dot{s}_{2}(t)=\varepsilon s_{1}+\left(a_{2}-2 s_{2}^{*}-\varepsilon\right) s_{2}-s_{2}^{2}, \\ \dot{I}_{1}(t)=\left(k s_{1}^{*}-b-c\right) I_{1}(t-\tau)+k s_{1} I_{1}(t-\tau),\end{array}\right.$ 
The linearization of Eq. (4) at $(0,0,0)$ is

$$
\left\{\begin{array}{l}
\dot{s}_{1}(t)=\left(a_{1}-2 s_{1}^{*}-\varepsilon\right) s_{1}+\varepsilon s_{2}-k s_{1}^{*} I_{1}(t-\tau), \\
\dot{s}_{2}(t)=\varepsilon s_{1}+\left(a_{2}-2 s_{2}^{*}-\varepsilon\right) s_{2} \\
\dot{I}_{1}(t)=\left(k s_{1}^{*}-b-c\right) I_{1}(t-\tau)
\end{array}\right.
$$

whose characteristic equation is

(6) $\left[\lambda-\left(k s_{1}^{*}-b-c\right) e^{-\lambda \tau}\right]\left\{\left[\lambda-\left(a_{1}-2 s_{1}^{*}-\varepsilon\right)\right]\left[\lambda-\left(a_{2}-2 s_{2}^{*}-\varepsilon\right)\right]-\varepsilon^{2}\right\}=0$.

Obviously, we have

$$
\lambda-\left(k s_{1}^{*}-b-c\right) e^{-\lambda \tau}=0
$$

or

$$
\left[\lambda-\left(a_{1}-2 s_{1}^{*}-\varepsilon\right)\right]\left[\lambda-\left(a_{2}-2 s_{2}^{*}-\varepsilon\right)\right]-\varepsilon^{2}=0 .
$$

In the following, we need to investigate the distribution of roots of Eq.(7) and Eq.(8). In order to investigate the distribution of roots of the transcendental equation Eq.(7) and Eq.(8), the following lemma is useful.

Lemma 2.1 ([5]). For the transcendental equation

$$
\begin{aligned}
P\left(\lambda, e^{-\lambda \tau_{1}}, \ldots, e^{-\lambda \tau_{m}}\right)= & \lambda^{n}+p_{1}^{(0)} \lambda^{n-1}+\cdots+p_{n-1}^{(0)} \lambda+p_{n}^{(0)} \\
& +\left[p_{1}^{(1)} \lambda^{n-1}+\cdots+p_{n-1}^{(1)} \lambda+p_{n}^{(1)}\right] e^{-\lambda \tau_{1}}+\cdots \\
& +\left[p_{1}^{(m)} \lambda^{n-1}+\cdots+p_{n-1}^{(m)} \lambda+p_{n}^{(m)}\right] e^{-\lambda \tau_{m}}=0
\end{aligned}
$$

as $\left(\tau_{1}, \tau_{2}, \tau_{3}, \ldots, \tau_{m}\right)$ vary, the sum of orders of the zeros of $P\left(\lambda, e^{-\lambda \tau_{1}}, \ldots, e^{-\lambda \tau_{m}}\right)$ in the open right half plane can change, and only a zero appears on or crosses the imaginary axis.

For $\tau=0,(7)$ deduces to

$$
\lambda=k s_{1}^{*}-b-c .
$$

It is easy to see that $\lambda=k s_{1}^{*}-b-c<0$ if the condition:

$$
k s_{1}^{*}<b+c
$$

holds.

For $\omega_{0}>0, i \omega_{0}$ is a root of $(7)$ if and only if

$$
i \omega_{0}=\left(k s_{1}^{*}-b-c\right)\left(\cos \omega_{0} \tau-i \sin \omega_{0} \tau\right) .
$$

Separating the real and imaginary parts, we get

$$
\left\{\begin{array}{l}
\left(k s_{1}^{*}-b-c\right) \cos \omega_{0} \tau=0 \\
\left(k s_{1}^{*}-b-c\right) \sin \omega_{0} \tau=-\omega_{0} .
\end{array}\right.
$$

Then we obtain

$$
\omega_{0}=-\left(k s_{1}^{*}-b-c\right), \tau_{k}=-\frac{1}{k s_{1}^{*}-b-c}\left(2 k \pi+\frac{\pi}{2}\right)(k=0,1,2, \ldots) .
$$


In the sequel, we consider the roots of Eq.(8). Obviously, (8) can be translated into the following form:

(12)

$$
\lambda^{2}-\left[a_{1}+a_{2}-2\left(s_{1}^{*}+s_{2}^{*}\right)-2 \varepsilon\right] \lambda+\left(a_{1}-2 s_{1}^{*}-\varepsilon\right)\left(a_{2}-2 s_{2}^{*}-\varepsilon\right)-\varepsilon^{2}=0 .
$$

A set of necessary and sufficient conditions for all roots of (12) to have a negative real part is given in the following form:

$$
a_{1}+a_{2}-2\left(s_{1}^{*}+s_{2}^{*}\right)-2 \varepsilon<0,\left(a_{1}-2 s_{1}^{*}-\varepsilon\right)\left(a_{2}-2 s_{2}^{*}-\varepsilon\right)-\varepsilon^{2}>0 .
$$

Let $\lambda(\tau)=\alpha(\tau)+i \omega(\tau)$ be a root of (7) near $\tau=\tau_{k}$, and $\alpha\left(\tau_{k}\right)=0$, and $\omega\left(\tau_{k}\right)=\omega_{0}$. Due to functional differential equation theory, for every $\tau_{k}, k=$ $0,1,2,3, \ldots$, there exists $\epsilon>0$ such that $\lambda(\tau)$ is continuously differentiable in $\tau$ for $\left|\tau-\tau_{k}\right|<\epsilon$. Substituting $\lambda(\tau)$ into the left hand of (7) and taking derivative with respect to $\tau$, we have

$$
\left[\frac{d \lambda}{d \tau}\right]^{-1}=-\frac{e^{\lambda \tau}}{\lambda\left(k s_{1}^{*}-b-c\right)}-\frac{\tau}{\lambda} .
$$

Then we obtain

$$
\left[\frac{d(\operatorname{Re} \lambda(\tau))}{d \tau}\right]_{\tau=\tau_{k}}^{-1}=-\frac{\sin \omega_{0} \tau_{k}}{\omega_{0}\left(k s_{1}^{*}-b-c\right)}>0 .
$$

The above analysis leads to the following results on the stability and Hopf bifurcation.

Theorem 2.2. If (H1)-(H3) hold, then the equilibrium $E_{0}\left(s_{1}^{*}, s_{2}^{*}, 0\right)$ of system (2) is asymptotically stable for $\tau \in\left[0, \tau_{0}\right)$ and unstable when $\tau>\tau_{0}$. Moreover, the system (2) undergoes a Hopf bifurcation at the boundary equilibrium $E_{0}\left(s_{1}^{*}, s_{2}^{*}, 0\right)$ when $\tau=\tau_{k}, k=0,1,2,3, \ldots$

\section{Direction and stability of the Hopf bifurcation}

In the previous section, we obtained conditions for Hopf bifurcation to occur when $\tau=\tau_{k}, k=0,1,2, \ldots$ In this section, we shall derive the explicit formulae determining the direction, stability, and period of these periodic solutions bifurcating from the boundary equilibrium $E_{0}\left(s_{1}^{*}, s_{2}^{*}, 0\right)$ at these critical value of $\tau$, by using techniques from normal form and center manifold theory [8]. Throughout this section, we always assume that the system (2) undergoes Hopf bifurcation at the boundary equilibrium $E_{0}\left(s_{1}^{*}, s_{2}^{*}, 0\right)$ for $\tau=\tau_{k}, k=0,1,2, \ldots$, and then $\pm i \omega_{0}$ is corresponding purely imaginary roots of the characteristic equation at the boundary equilibrium $E_{0}\left(s_{1}^{*}, s_{2}^{*}, 0\right)$.

For convenience, let $\bar{s}_{i}(t)=s_{i}(\tau t)(i=1,2)$ and $\bar{I}_{1}(t)=I_{1}(\tau t), \tau=\tau_{k}+\mu$, where $\tau_{k}$ is defined by (11) and $\mu \in R$, drop the bar for the simplification of notations, then the system (4) can be written as an FDE in $\left.C=C([-1,0]), R^{3}\right)$ as

$$
\dot{u}(t)=L_{\mu}\left(u_{t}\right)+F\left(\mu, u_{t}\right),
$$


where $u(t)=\left(s_{1}(\tau t), s_{2}(\tau t), I_{1}(\tau t)\right)^{T} \in C$ and $u_{t}(\theta)=u(t+\theta)=\left(s_{1}(t+\right.$ $\left.\theta), s_{2}(t+\theta), I_{1}(t+\theta)\right)^{T} \in C$, and $L_{\mu}: C \rightarrow R, F: R \times C \rightarrow R$ are given by

$$
\begin{aligned}
L_{\mu} \phi= & \left(\tau_{k}+\mu\right)\left(\begin{array}{ccc}
a_{1}-2 s_{1}^{*}-\varepsilon & \varepsilon & 0 \\
\varepsilon & a_{2}-2 s_{2}^{*}-\varepsilon & 0 \\
0 & 0 & 0
\end{array}\right)\left(\begin{array}{l}
\phi_{1}(0) \\
\phi_{2}(0) \\
\phi_{3}(0)
\end{array}\right) \\
& +\left(\tau_{k}+\mu\right)\left(\begin{array}{ccc}
0 & 0 & -k s_{1}^{*} \\
0 & 0 & 0 \\
0 & 0 & k s_{1}^{*}-b-c
\end{array}\right)\left(\begin{array}{c}
\phi_{1}(-1) \\
\phi_{2}(-1) \\
\phi_{3}(-1)
\end{array}\right)
\end{aligned}
$$

and

$$
F(\mu, \phi)=\left(\tau_{k}+\mu\right)\left(\begin{array}{c}
-\phi_{1}^{2}(0)-k \phi_{1}(0) \phi_{3}(-1) \\
-\phi_{2}^{2}(0) \\
k \phi_{1}(0) \phi_{3}(-1)
\end{array}\right),
$$

respectively, where $\phi(\theta)=\left(\phi_{1}(\theta), \phi_{2}(\theta), \phi_{3}(\theta)\right)^{T} \in C$.

From the discussion in Section 2, we know that if $\mu=0$, then the system (3.1) undergoes a Hopf bifurcation at the boundary equilibrium $E_{0}\left(s_{1}^{*}, s_{2}^{*}, 0\right)$ and the associated characteristic equation of the system (15) has a pair of simple imaginary roots $\pm \omega_{0} \tau_{k}$.

By the representation theorem, there is a matrix function with bounded variation components $\eta(\theta, \mu), \theta \in[-1,0]$ such that

$$
L_{\mu} \phi=\int_{-1}^{0} d \eta(\theta, \mu) \phi(\theta) \text { for } \phi \in C .
$$

In fact, we can choose

$$
\begin{aligned}
\eta(\theta, \mu)= & \left(\tau_{k}+\mu\right)\left(\begin{array}{ccc}
a_{1}-2 s_{1}^{*}-\varepsilon & \varepsilon & 0 \\
\varepsilon & a_{2}-2 s_{2}^{*}-\varepsilon & 0 \\
0 & 0 & 0
\end{array}\right) \delta(\theta) \\
& -\left(\tau_{k}+\mu\right)\left(\begin{array}{ccc}
0 & 0 & -k s_{1}^{*} \\
0 & 0 & 0 \\
0 & 0 & k s_{1}^{*}-b-c
\end{array}\right) \delta(\theta+1),
\end{aligned}
$$

where $\delta$ is the Dirac delta function.

For $\phi \in C\left([-1,0], R^{3}\right)$, define

$$
A(\mu) \phi=\left\{\begin{array}{lc}
\frac{d \phi(\theta)}{d \theta}, & -1 \leq \theta<0, \\
\int_{-1}^{0} d \eta(s, \mu) \phi(s), & \theta=0
\end{array}\right.
$$

and

$$
R \phi=\left\{\begin{array}{lr}
0, & -1 \leq \theta<0 \\
F(\mu, \phi), & \theta=0
\end{array}\right.
$$

Then (15) is equivalent to the abstract differential equation

$$
\dot{u}_{t}=A(\mu) u_{t}+R(\mu) u_{t}
$$

where $u_{t}(\theta)=u(t+\theta), \theta \in[-1,0]$. 
For $\psi \in C\left([-1,0],\left(R^{3}\right)^{*}\right)$, define

$$
A^{*} \psi(s)=\left\{\begin{array}{lc}
-\frac{d \psi(s)}{d s}, & s \in(0,1], \\
\int_{-1}^{0} d \eta^{T}(t, 0) \psi(-t), & s=0 .
\end{array}\right.
$$

For $\phi \in C\left([-1,0], R^{3}\right)$ and $\psi \in C\left([0,1],\left(R^{3}\right)^{*}\right)$, define the bilinear form

$$
\langle\psi, \phi\rangle=\bar{\psi}(0) \phi(0)-\int_{-1}^{0} \int_{\xi=0}^{\theta} \psi^{T}(\xi-\theta) d \eta(\theta) \phi(\xi) d \xi,
$$

where $\eta(\theta)=\eta(\theta, 0)$, the $A=A(0)$ and $A^{*}$ are adjoint operators. By the discussions in Section 2, we know that $\pm i \omega_{0} \tau_{k}$ are eigenvalues of $A(0)$, and they are also eigenvalues of $A^{*}$ corresponding to $i \omega_{0} \tau_{k}$ and $-i \omega_{0} \tau_{k}$ respectively. By direct computation, we can obtain

$$
q(\theta)=(1, \alpha, \beta)^{T} e^{i \omega_{0} \tau_{k} \theta}, q^{*}(s)=M\left(1, \alpha^{*}, \beta^{*}\right) e^{i \omega_{0} \tau_{k} s}, M=\frac{1}{B},
$$

where

$$
\begin{aligned}
\alpha & =\frac{\varepsilon}{i \omega_{0}-\left(a_{2}-2 s_{2}^{*}-\varepsilon\right)}, \beta=\frac{\varepsilon \alpha-i \omega_{0}+a_{1}-2 s_{1}^{*}-\varepsilon}{k s_{1}^{*} e^{-i \omega_{0} \tau_{k}}}, \\
\alpha^{*} & =-\frac{i \omega_{0}+a_{1}-2 s_{1}^{*}-\varepsilon}{\varepsilon}, \beta^{*}=\frac{k s_{1}^{*}}{i \omega_{0}+k s_{1}^{*}-b-c}, \\
B & =1+\bar{\alpha} \alpha^{*}+\bar{\beta} \beta^{*}+\tau_{k}\left[\bar{\alpha} \beta^{*}\left(k s_{1}^{*}-b-c\right)-k s_{1}^{*} \bar{\alpha}\right] .
\end{aligned}
$$

Furthermore, $\left\langle q^{*}(s), q(\theta)\right\rangle=1$ and $\left\langle q^{*}(s), \bar{q}(\theta)\right\rangle=0$.

Next, we use the same notations as those in Hassard [8] and we first compute the coordinates to describe the center manifold $C_{0}$ at $\mu=0$. Let $u_{t}$ be the solution of Eq.(15) when $\mu=0$.

Define

$$
z(t)=\left\langle q^{*}, u_{t}\right\rangle, W(t, \theta)=u_{t}(\theta)-2 \operatorname{Re}\{z(t) q(\theta)\} .
$$

on the center manifold $C_{0}$, and we have

$$
W(t, \theta)=W(z(t), \bar{z}(t), \theta)
$$

where

$$
W(z(t), \bar{z}(t), \theta)=W(z, \bar{z})=W_{20} \frac{z^{2}}{2}+W_{11} z \bar{z}+W_{02} \frac{\bar{z}^{2}}{2}+\cdots,
$$

and $z$ and $\bar{z}$ are local coordinates for center manifold $C_{0}$ in the direction of $q^{*}$ and $\bar{q}^{*}$. Noting that $W$ is also real if $u_{t}$ is real, we consider only real solutions. For solutions $u_{t} \in C_{0}$ of (15),

$$
\dot{z}(t)=i \omega_{0} \tau_{k} z+\bar{q}^{*}(\theta) f\left(0, W(z, \bar{z}, \theta)+2 \operatorname{Re}\{z q(\theta)\} \stackrel{\text { def }}{=} i \omega_{0} \tau_{k} z+\bar{q}^{*}(0) f_{0} .\right.
$$

That is

where

$$
\dot{z}(t)=i \omega_{0} \tau_{k} z+g(z, \bar{z})
$$

$$
g(z, \bar{z})=g_{20} \frac{z^{2}}{2}+g_{11} z \bar{z}+g_{02} \frac{\bar{z}^{2}}{2}+\cdots .
$$


Hence, we have

$g(z, \bar{z})=\bar{q}^{*}(0) f_{0}(z, \bar{z})=f\left(0, y_{t}\right)=K_{20} z^{2}+K_{11} z \bar{z}+K_{02} \bar{z}^{2}+K_{21} z^{2} \bar{z}+$ h.o.t., where

$$
\begin{aligned}
K_{20}= & -\bar{D} \tau_{k}\left(1+k \beta e^{-i \omega_{0} \tau_{k}}+\alpha^{2} \alpha^{*}-k \beta \beta^{*} e^{-i \omega_{0} \tau_{k}}\right), \\
K_{11}= & -2 \bar{D} \tau_{k}\left(1+k \operatorname{Re}\left\{\beta e^{-i \omega_{0} \tau_{k}}\right\}+|\alpha|^{2} \alpha^{*}-k \beta^{*} \operatorname{Re}\left\{\beta e^{-i \omega_{0} \tau_{k}}\right\}\right), \\
K_{02}= & -\bar{D} \tau_{k}\left(1+k \bar{\beta} e^{i \omega_{0} \tau_{k}}+\bar{\alpha}^{2} \alpha^{*}-k \bar{\beta} \beta^{*} e^{i \omega_{0} \tau_{k}}\right), \\
K_{21}= & -\bar{D} \tau_{k}\left\{W_{20}^{(1)}(0)+2 W_{11}^{(1)}(0)+k\left[\frac{1}{2} \bar{\beta} W_{20}^{(1)}(0) e^{i \omega_{0} \tau_{k}}+\frac{1}{2} W_{20}^{(3)}(-1)\right.\right. \\
& \left.+\beta W_{11}^{(1)}(0) e^{-i \omega_{0} \tau_{k}}+W_{11}^{(3)}(-1)\right]+\alpha^{*}\left[\bar{\alpha} W_{20}^{(1)}(0)+2 \alpha W_{11}^{(1)}(0)\right] \\
& \left.+k \beta^{*}\left[\frac{1}{2} \bar{\beta} W_{20}^{(1)}(0) e^{i \omega_{0} \tau_{k}}+\frac{1}{2} W_{20}^{(3)}(-1)+\beta W_{11}^{(1)}(0) e^{-i \omega_{0} \tau_{k}}+W_{11}^{(3)}(-1)\right]\right\} .
\end{aligned}
$$

Then we obtain

$$
g_{20}=2 K_{20}, g_{11}=K_{11}, g_{02}=2 K_{02}, g_{21}=2 K_{21} .
$$

For unknown $W_{20}^{(1)}(0), W_{20}^{(3)}(-1), W_{11}^{(1)}(0), W_{11}^{(3)}(-1)$ in $g_{21}$, we still need to compute them.

From (22), (23), we have

(26)

$$
W^{\prime}=\left\{\begin{array}{l}
A W-2 \operatorname{Re}\left\{\bar{q}^{*}(0) \bar{f} q(\theta)\right\}, \\
A W-2 \operatorname{Re}\left\{\bar{q}^{*}(0) \bar{f} q(\theta)\right\}+\bar{f}, \\
A=0
\end{array} \quad \stackrel{\text { def }}{=} A W+H(z, \bar{z}, \theta),\right.
$$

where

$$
H(z, \bar{z}, \theta)=H_{20}(\theta) \frac{z^{2}}{2}+H_{11}(\theta) z \bar{z}+H_{02}(\theta) \frac{\bar{z}^{2}}{2}+\cdots
$$

Comparing the coefficients, we obtain

$$
\begin{gathered}
\left(A W-2 i \tau_{k} \omega_{0}\right) W_{20}=-H_{20}(\theta), \\
A W_{11}(\theta)=-H_{11}(\theta),
\end{gathered}
$$

And we know that for $\theta \in[-1,0)$,

(30) $H(z, \bar{z}, \theta)=-\bar{q}^{*}(0) f_{0} q(\theta)-q^{*}(0) \bar{f}_{0} \bar{q}(\theta)=-g(z, \bar{z}) q(\theta)-\bar{g}(z, \bar{z}) \bar{q}(\theta)$.

Comparing the coefficients of (30) with (27) gives that

$$
\begin{aligned}
& H_{20}(\theta)=-g_{20} q(\theta)-\bar{g}_{02} \bar{q}(\theta), \\
& H_{11}(\theta)=-g_{11} q(\theta)-\bar{g}_{11} \bar{q}(\theta) .
\end{aligned}
$$

From $(28),(31)$ and the definition of $A$, we get

$$
\dot{W}_{20}(\theta)=2 i \omega_{0} \tau_{k} W_{20}(\theta)+g_{20} q(\theta)+g_{02} \bar{q}(\theta) .
$$


Noting that $q(\theta)=q(0) e^{i \omega_{0} \tau_{k} \theta}$, we have

$$
W_{20}(\theta)=\frac{i g_{20}}{\omega_{0} \tau_{k}} q(0) e^{i \omega_{0} \tau_{k} \theta}+\frac{i \bar{g}_{02}}{3 \omega_{0} \tau_{k}} \bar{q}(0) e^{-i \omega_{0} \tau_{k} \theta}+E_{1} e^{2 i \omega_{0} \tau_{k} \theta},
$$

where $E_{1}=\left(E_{1}^{(1)}, E_{1}^{(2)}, E_{1}^{(3)}\right) \in R^{3}$ is a constant vector.

Similarly, from (29), (32) and the definition of $A$, we have

$$
\begin{gathered}
\dot{W}_{11}(\theta)=g_{11} q(\theta)+g_{11}^{-} \bar{q}(\theta), \\
W_{11}(\theta)=-\frac{i g_{11}}{\omega_{0} \tau_{k}} q(0) e^{i \omega_{0} \tau_{k} \theta}+\frac{i \bar{g}_{11}}{\omega_{0} \tau_{k}} \bar{q}(0) e^{-i \omega_{0} \tau_{k} \theta}+E_{2},
\end{gathered}
$$

where $E_{2}=\left(E_{2}^{(1)}, E_{2}^{(2)}, E_{2}^{(3)}\right) \in R^{3}$ is a constant vector.

In what follows, we shall seek appropriate $E_{1}, E_{2}$ in (34), (36), respectively. It follows from the definition of $A$ and (31), (32) that

$$
\int_{-1}^{0} d \eta(\theta) W_{20}(\theta)=2 i \omega_{0} \tau_{k} W_{20}(0)-H_{20}(0)
$$

and

$$
\int_{-1}^{0} d \eta(\theta) W_{11}(\theta)=-H_{11}(0)
$$

where $\eta(\theta)=\eta(0, \theta)$.

From (28), we have

$$
\begin{gathered}
H_{20}(0)=-g_{20} q(0)-g_{02}^{-} \bar{q}(0)+2 \tau_{k}\left(H_{1}, H_{2}, H_{3}\right)^{T}, \\
H_{11}(0)=-g_{11} q(0)-g_{11}^{\overline{1}}(0) \bar{q}(0)+2 \tau_{k}\left(P_{1}, P_{2}, P_{3}\right)^{T},
\end{gathered}
$$

where

$$
\begin{aligned}
& H_{1}=-1-k \beta e^{-i \omega_{0} \tau_{k}}, H_{2}=\alpha^{2}, H_{3}=k \beta e^{-i \omega_{0} \tau_{k}}, \\
& P_{1}=-1-k \operatorname{Re}\left\{\beta e^{-i \omega_{0} \tau_{k}}\right\}, P_{2}=|\alpha|^{2}, P_{3}=k \operatorname{Re}\left\{\bar{\beta} e^{-i \omega_{0} \tau_{k}}\right\} .
\end{aligned}
$$

Noting that

$$
\begin{gathered}
\left(i \omega_{0} \tau_{k} I-\int_{-1}^{0} e^{i \omega_{0} \tau_{k} \theta} d \eta(\theta)\right) q(0)=0 \\
\left(-i \omega_{0} \tau_{k} I-\int_{-1}^{0} e^{-i \omega_{0} \tau_{k} \theta} d \eta(\theta)\right) \bar{q}(0)=0
\end{gathered}
$$

and substituting (34) and (39) into (37), we have

$$
\left(2 i \omega_{0} \tau_{k} I-\int_{-1}^{0} e^{2 i \omega_{0} \tau_{k} \theta} d \eta(\theta)\right) E_{1}=2 \tau_{k}\left(H_{1}, H_{2}, H_{3}\right)^{T} .
$$

That is,

$$
\begin{aligned}
& \left(\begin{array}{ccc}
2 i \omega_{0}-\left(a_{1}-2 s_{1}^{*}-\varepsilon\right) & -\varepsilon & -k s_{1}^{*} e^{-2 i \omega_{0} \tau_{k}} \\
-\varepsilon & 2 i \omega_{0}-\left(a_{2}-2 s_{2}^{*}-\varepsilon\right) & 0 \\
0 & 0 & 2 i \omega_{0}-\left(k s_{1}^{*}-b-c\right) e^{-2 i \omega_{0} \tau_{k}}
\end{array}\right) \times E_{1} \\
& =2\left(H_{1}, H_{2}, H_{3}\right)^{T} \text {. }
\end{aligned}
$$


It follows that

$$
E_{1}^{(1)}=\frac{\Delta_{11}}{\Delta_{1}}, E_{1}^{(2)}=\frac{\Delta_{12}}{\Delta_{1}}, E_{1}^{(2)}=\frac{\Delta_{13}}{\Delta_{1}},
$$

where

$$
\begin{aligned}
& \Delta_{1}=\operatorname{det}\left(\begin{array}{ccc}
2 i \omega_{0}-\left(a_{1}-2 s_{1}^{*}-\varepsilon\right) & -\varepsilon & -k s_{1}^{*} e^{-2 i \omega_{0} \tau_{k}} \\
-\varepsilon & 2 i \omega_{0}-\left(a_{2}-2 s_{2}^{*}-\varepsilon\right) & 0 \\
0 & 0 & 2 i \omega_{0}-\left(k s_{1}^{*}-b-c\right) e^{-2 i \omega_{0} \tau_{k}}
\end{array}\right) \text {, } \\
& \Delta_{11}=2 \operatorname{det}\left(\begin{array}{ccc}
H_{1} & -\varepsilon & -k s_{1}^{*} e^{-2 i \omega_{0} \tau_{k}} \\
H_{2} & 2 i \omega_{0}-\left(a_{2}-2 s_{2}^{*}-\varepsilon\right) & 0 \\
H_{3} & 0 & 2 i \omega_{0}-\left(k s_{1}^{*}-b-c\right) e^{-2 i \omega_{0} \tau_{k}}
\end{array}\right) \text {, } \\
& \Delta_{12}=2 \operatorname{det}\left(\begin{array}{ccc}
2 i \omega_{0}-\left(a_{1}-2 s_{1}^{*}-\varepsilon\right) & H_{1} & -k s_{1}^{*} e^{-2 i \omega_{0} \tau_{k}} \\
-\varepsilon & H_{2} & 0 \\
0 & H_{3} & 2 i \omega_{0}-\left(k s_{1}^{*}-b-c\right) e^{-2 i \omega_{0} \tau_{k}}
\end{array}\right) \text {, } \\
& \Delta_{13}=2 \operatorname{det}\left(\begin{array}{ccc}
2 i \omega_{0}-\left(a_{1}-2 s_{1}^{*}-\varepsilon\right) & -\varepsilon & H_{1} \\
-\varepsilon & 2 i \omega_{0}-\left(a_{2}-2 s_{2}^{*}-\varepsilon\right) & H_{2} \\
0 & 0 & H_{3}
\end{array}\right) \text {. }
\end{aligned}
$$

Similarly, substituting (35) and (40) into (38), we have

$$
\left(\int_{-1}^{0} d \eta(\theta)\right) E_{2}=2 \tau_{k}\left(P_{1}, P_{2}, P_{3}\right)^{T} \text {. }
$$

That is

$$
\left(\begin{array}{ccc}
a_{1}-2 s_{1}^{*}-\varepsilon & \varepsilon & -k s_{1}^{*} \\
\varepsilon & a_{2}-2 s_{2}^{*}-\varepsilon & 0 \\
0 & 0 & k s_{1}^{*}-b-c
\end{array}\right) E_{2}=2\left(-P_{1},-P_{2},-P_{3}\right)^{T} .
$$

It follows that

$$
E_{2}^{(1)}=\frac{\Delta_{21}}{\Delta_{2}}, E_{2}^{(2)}=\frac{\Delta_{22}}{\Delta_{2}}, E_{2}^{(2)}=\frac{\Delta_{23}}{\Delta_{2}},
$$

where

$$
\begin{aligned}
& \Delta_{2}=\operatorname{det}\left(\begin{array}{ccc}
a_{1}-2 s_{1}^{*}-\varepsilon & \varepsilon & -k s_{1}^{*} \\
\varepsilon & a_{2}-2 s_{2}^{*}-\varepsilon & 0 \\
0 & 0 & k s_{1}^{*}-b-c
\end{array}\right), \\
& \Delta_{21}=2 \operatorname{det}\left(\begin{array}{ccc}
-P_{1} & \varepsilon & -k s_{1}^{*} \\
-P_{2} & a_{2}-2 s_{2}^{*}-\varepsilon & 0 \\
-P_{3} & 0 & k s_{1}^{*}-b-c
\end{array}\right), \\
& \Delta_{22}=2 \operatorname{det}\left(\begin{array}{ccc}
a_{1}-2 s_{1}^{*}-\varepsilon & -P_{1} & -k s_{1}^{*} \\
\varepsilon & -P_{2} & 0 \\
0 & -P_{3} & k s_{1}^{*}-b-c
\end{array}\right), \\
& \Delta_{23}=2 \operatorname{det}\left(\begin{array}{ccc}
a_{1}-2 s_{1}^{*}-\varepsilon & \varepsilon & -P_{1} \\
\varepsilon & a_{2}-2 s_{2}^{*}-\varepsilon & -P_{2} \\
0 & 0 & -P_{3}
\end{array}\right) .
\end{aligned}
$$


From (34), (36), (41), (42), we can calculate $g_{21}$ and derive the following values:

$$
\begin{aligned}
c_{1}(0) & =\frac{i}{2 \omega_{0} \tau_{k}}\left(g_{20} g_{11}-2\left|g_{11}\right|^{2}-\frac{\left|g_{02}\right|^{2}}{3}\right)+\frac{g_{21}}{2} \\
\mu_{2} & =-\frac{\operatorname{Re}\left\{c_{1}(0)\right\}}{\operatorname{Re}\left\{\lambda^{\prime}\left(\tau_{k}\right\}\right.} \\
\beta_{2} & =2 \operatorname{Re}\left(c_{1}(0)\right), \\
T_{2} & =-\frac{\operatorname{Im}\left\{c_{1}(0)\right\}+\mu_{2} \operatorname{Im}\left\{\lambda^{\prime}\left(\tau_{k}\right)\right\}}{\omega_{0} \tau_{k}} .
\end{aligned}
$$

These formulaes give a description of the Hopf bifurcation periodic solutions of (15) at $\tau=\tau_{k},(k=0,1,2,3, \ldots)$ on the center manifold. From the discussion above, we have the following result:

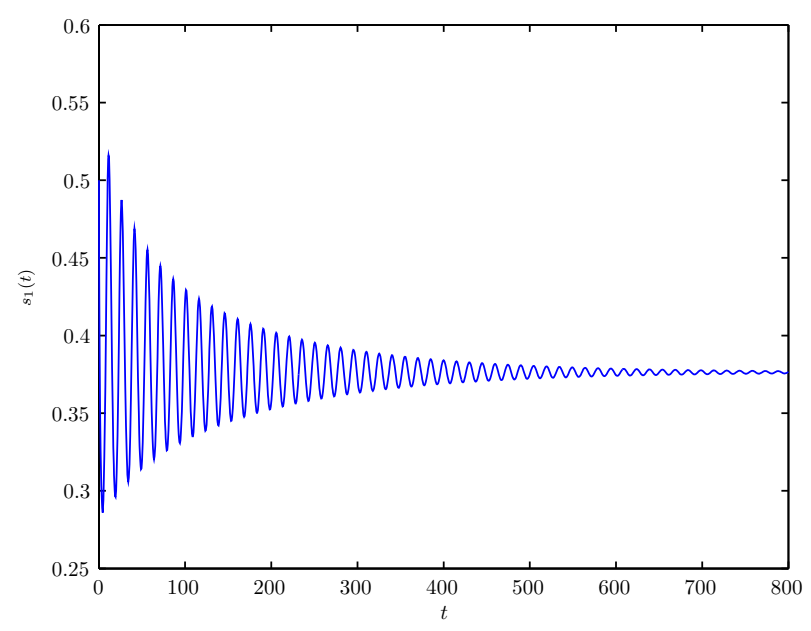

Figure 1

Theorem 3.1. The periodic solution is supercritical (subcritical) if $\mu_{2}>0$ $\left(\mu_{2}<0\right)$; the bifurcating periodic solutions are orbitally asymptotically stable with asymptotical phase (unstable) if $\beta_{2}<0\left(\beta_{2}>0\right)$; the periodic of the bifurcating periodic solutions increase (decrease) if $T_{2}>0\left(T_{2}<0\right)$.

Remark 3.2. A $\tau T$-periodic solution of (15) is a $T$-periodic solution of (4).

\section{Numerical examples}

In this section, we present some numerical results of the system (2) to verify the analytical predictions obtained in the previous section. From Section 3, 


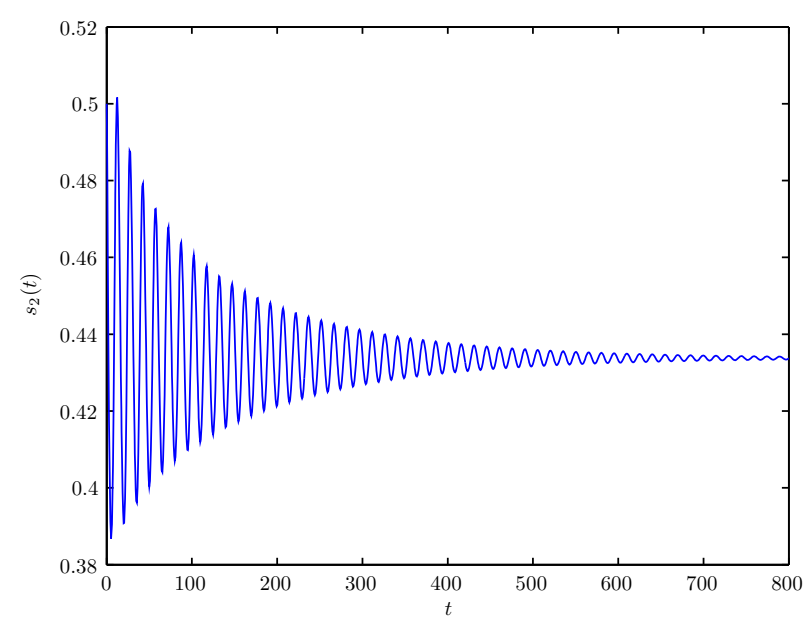

Figure 2

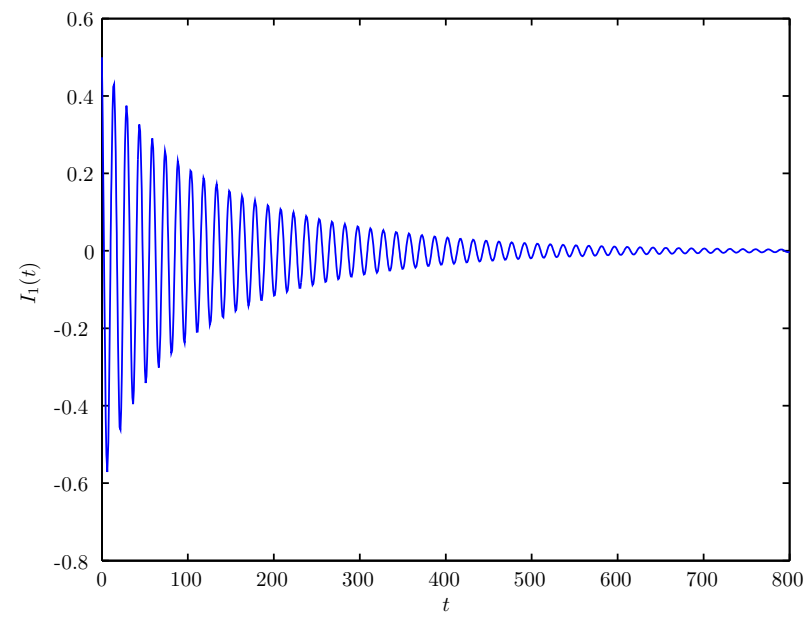

FiguRe 3

we may determine the direction of a Hopf bifurcation and the stability of the bifurcation periodic solutions. Let us consider the following system:

$$
\left\{\begin{array}{l}
\dot{s}_{1}=-0.5 s_{1} I_{1}(t-\tau)+s_{1}\left(0.3-s_{1}\right)+0.5\left(s_{2}-s_{1}\right), \\
\dot{s}_{2}=s_{2}\left(0.5-s_{2}\right)+0.5\left(s_{1}-s_{2}\right), \\
\dot{I}_{1}=0.5 s_{1} I_{1}(t-\tau)-0.2 I_{1}(t-\tau)-0.4 I_{1}(t-\tau)
\end{array}\right.
$$

which has a boundary equilibrium $E_{0}\left(s_{1}^{*}, s_{2}^{*}, I_{1}^{*}\right) \approx(0.3632,0.4262,0)$ and satisfies the conditions indicated in Theorem 2.2. When $\tau=0$, the boundary 


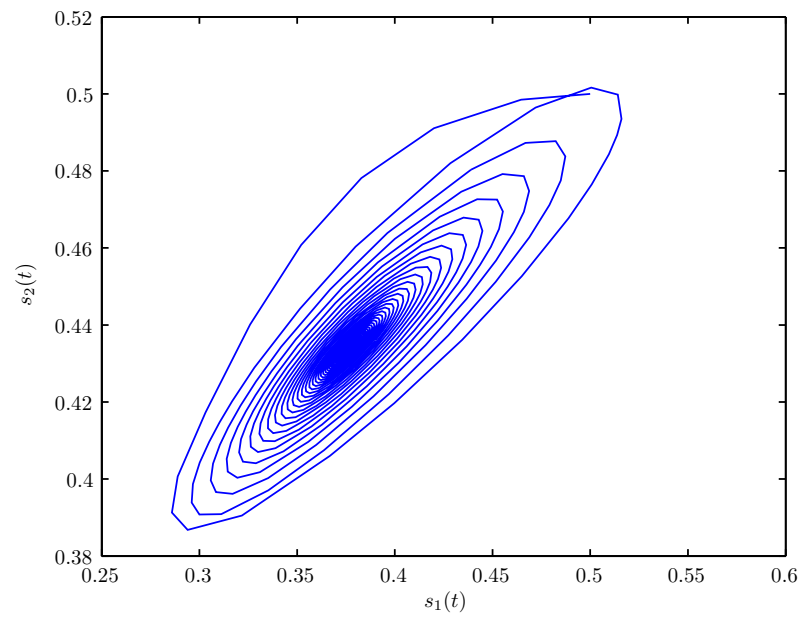

Figure 4

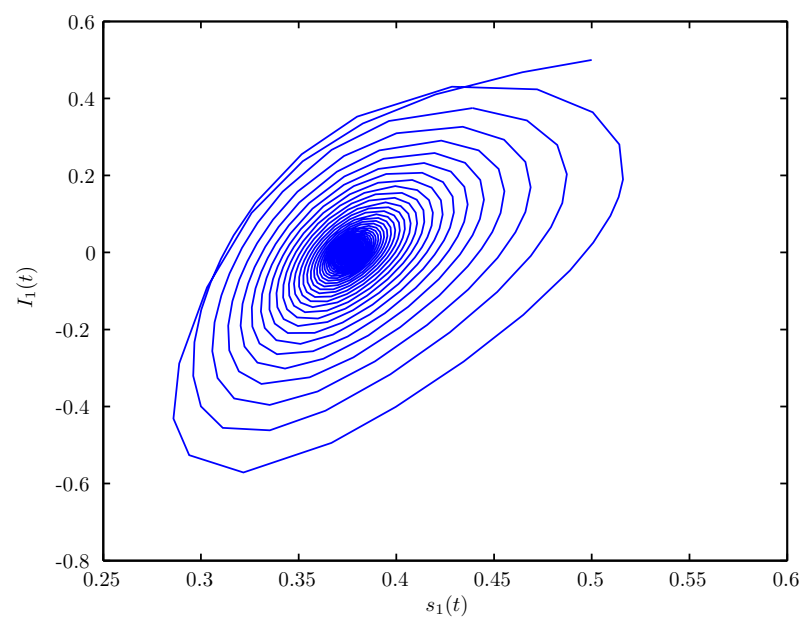

Figure 5

equilibrium $E_{0} \approx(0.3632,0.4262,0)$ is asymptotically stable. Take $k=0$ for example, by some complicated computation by means of Matlab 7.0, we get $\omega_{0} \approx 1.0328, \tau_{0} \approx 3.7544, \lambda^{\prime}\left(\tau_{0}\right) \approx 0.3345-6.6612 i$. Thus we can calculate the following values: $c_{1}(0) \approx-1.0531-3.4312 i, \mu_{2} \approx 0.4355, \beta_{2} \approx-4.0564, T_{2} \approx$ 5.4005. Furthermore, it follows that $\mu_{2}>0$ and $\beta_{2}<0$. Thus, the boundary equilibrium $E_{0} \approx(0.3632,0.4262,0)$ is stable when $\tau<\tau_{0}$ as is illustrated by the computer simulations (see Fig.1-7). When $\tau$ passes through the critical value $\tau_{0}$, the boundary equilibrium $E_{0} \approx(0.3632,0.4262,0)$ loses its stability 


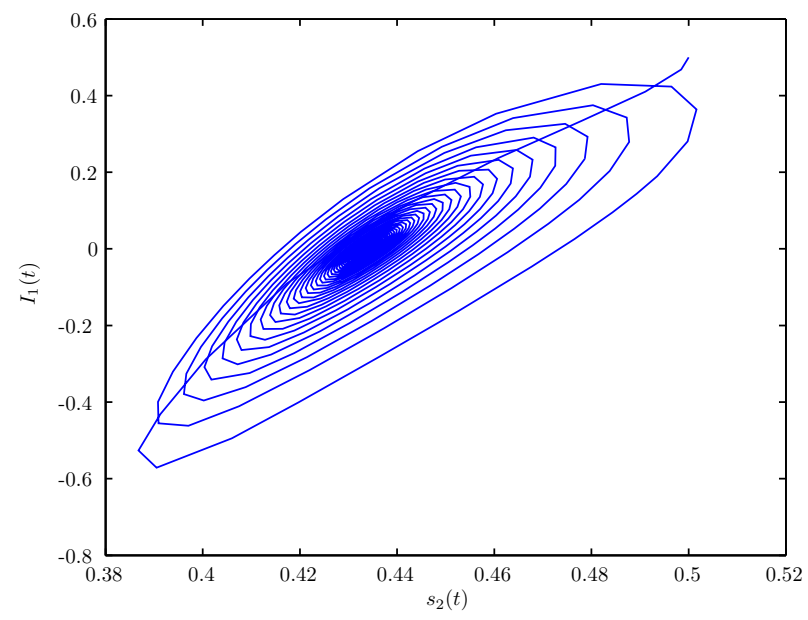

Figure 6

and a Hopf bifurcation occurs, i.e., a family of periodic solutions bifurcations from the boundary equilibrium $E_{0} \approx(0.3632,0.4262,0)$. Since $\mu_{2}>0$ and $\beta_{2}<0$, the direction of the Hopf bifurcation is $\tau>\tau_{0}$, and these bifurcating periodic solutions from $E_{0} \approx(0.3632,0.4262,0)$ at $\tau_{0}$ are stable, which are depicted in Fig.8-14.

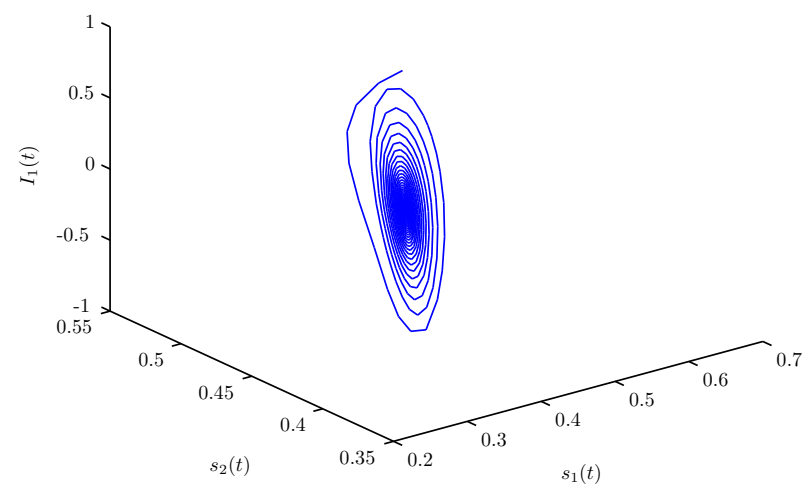

FiguRE 7

Fig.1-Fig.7 Behavior and phase portrait of the system (43) with $\tau=3.7<$ $\tau_{0} \approx 3.7544$. The boundary equilibrium $E_{0} \approx(0.3632,0.4262,0)$ is asymptotically stable. The initial value is $(0.5,0.5,0.5)$.

Fig.8-Fig.14 Behavior and phase portrait of the system (43) with $\tau=4>$ $\tau_{0} \approx 3.7544$. Hopf bifurcation occurs from the boundary equilibrium $E_{0} \approx$ $(0.3632,0.4262,0)$. The initial value is $(0.5,0.5,0.5)$. 


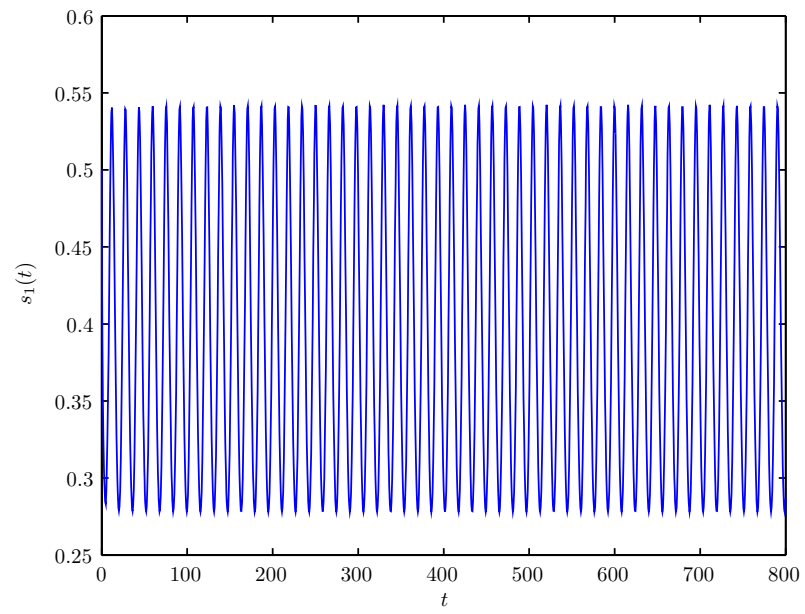

FIGURE 8

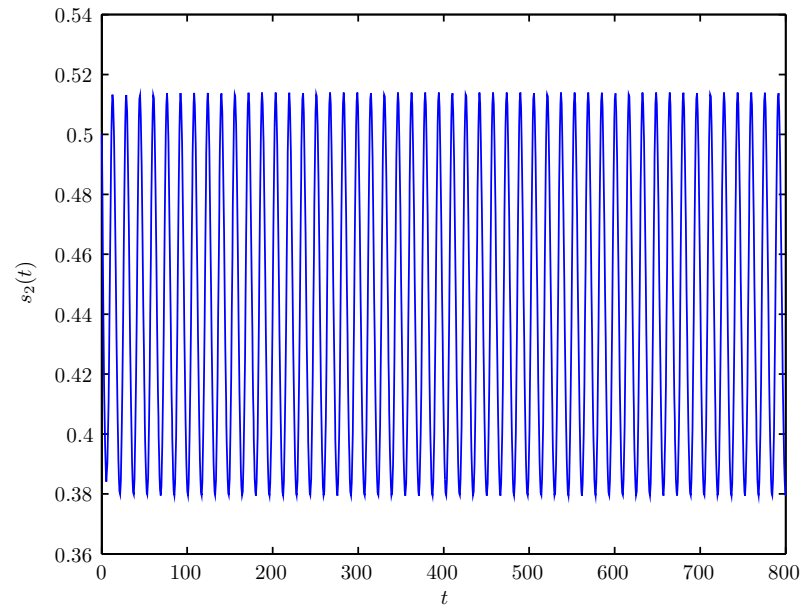

FIGURE 9

\section{Biological explanations and conclusions}

\subsection{Biological explanations}

From the analysis in Section 2, we know that under the conditions (H1)-(H3), then the boundary equilibrium $E_{0}\left(s_{1}^{*}, s_{2}^{*}, 0\right)$ of the system (2) is asymptotically stable for all $\tau \in\left[0, \tau_{0}\right)$. This shows that, in this case, the susceptible numbers in place 1 , the susceptible numbers in place 2 and the infective numbers in place 1 will tend to stabilization, that is, the susceptible numbers in place 1 , the susceptible numbers in place 2 and the infective numbers in place 1 will 


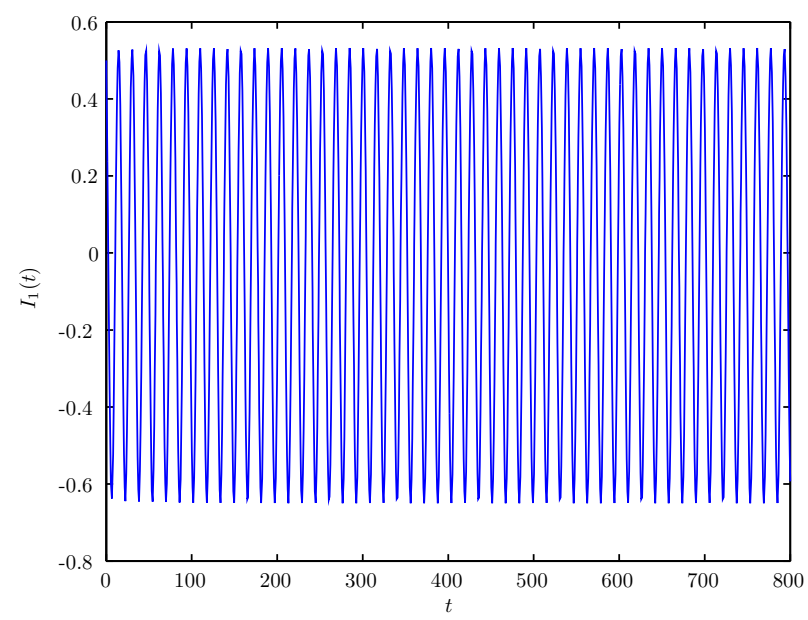

FIGURE 10

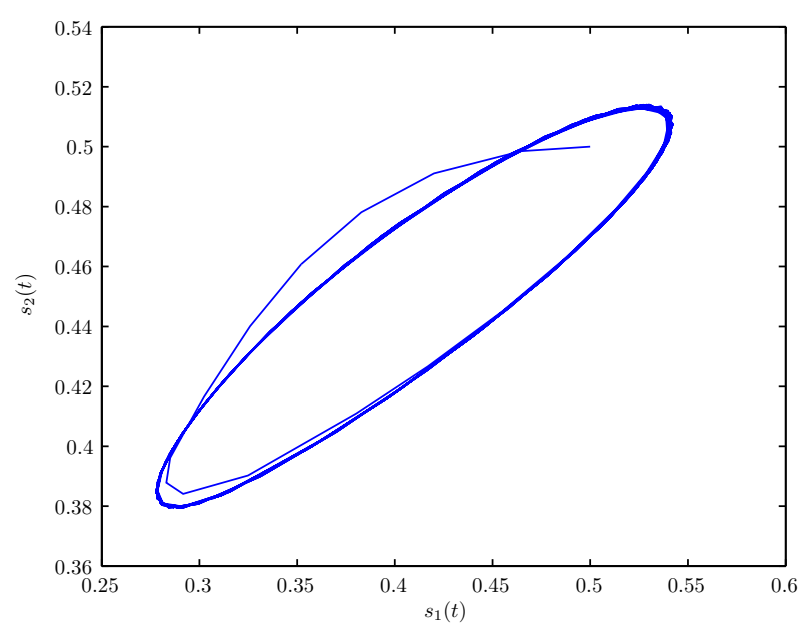

FIGURE 11

tend to $s_{1}^{*}, s_{2}^{*}, 0$, respectively, and this fact is not influenced by the delay $\tau \in$ $\left[0, \tau_{0}\right)$. When $\tau$ crosses through the critical value $\tau_{0}$, the boundary equilibrium $E_{0}\left(s_{1}^{*}, s_{2}^{*}, 0\right)$ of the system (2) loses stability and a Hopf bifurcation occurs. This shows that the susceptible numbers in place 1 , the susceptible numbers in place 2 and the infective numbers in place 1 keep in an oscillatory mode near the boundary equilibrium $E_{0}\left(s_{1}^{*}, s_{2}^{*}, 0\right)$.

\subsection{Conclusions}




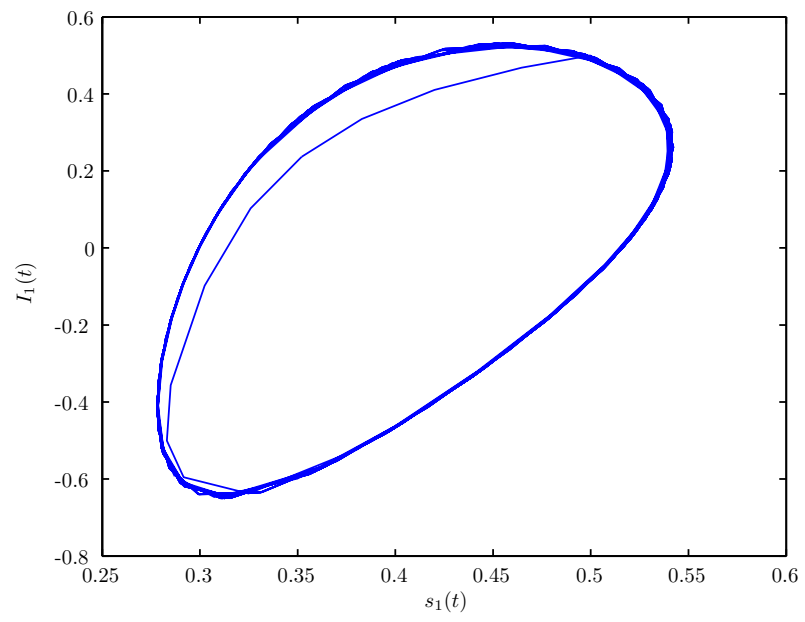

Figure 12

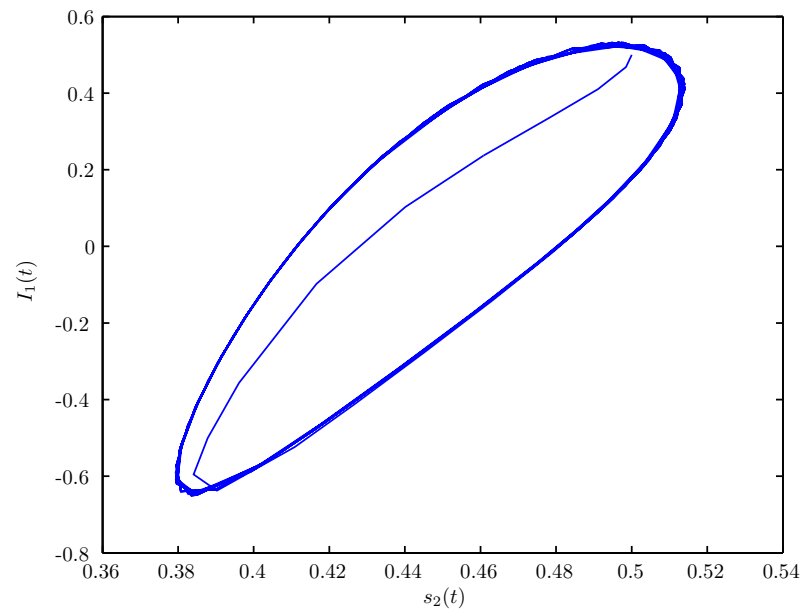

FIGURE 13

In this paper, we have investigated local stability of the boundary equilibrium $E_{0}\left(s_{1}^{*}, s_{2}^{*}, 0\right)$ and local Hopf bifurcation in delayed epidemic model with diffusion. We have showed that if the conditions (H1)-(H3) hold, the boundary equilibrium $E_{0}\left(s_{1}^{*}, s_{2}^{*}, 0\right)$ of the system $(2)$ is asymptotically stable for all $\tau \in\left[0, \tau_{0}\right)$. As the delay $\tau$ increases, the boundary equilibrium loses its stability and a sequence of Hopf bifurcations occur at the boundary equilibrium $E_{0}\left(s_{1}^{*}, s_{2}^{*}, 0\right)$, i.e., a family of periodic orbits bifurcates from the the positive 


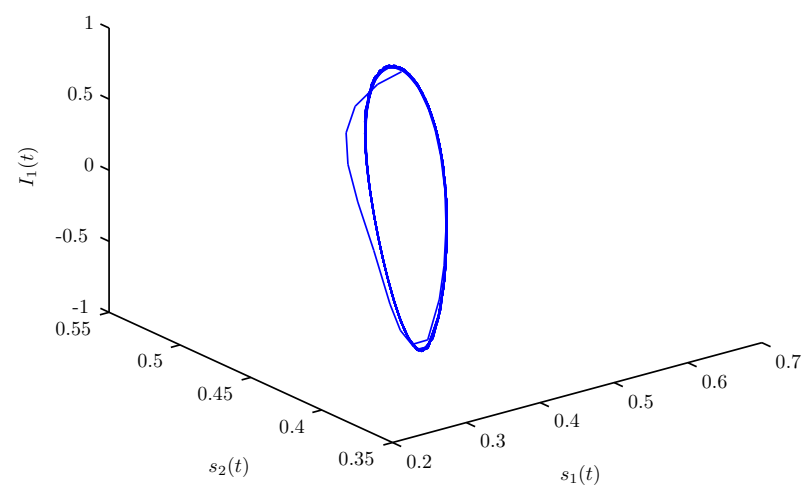

FiguRe 14

equilibrium $E_{0}\left(s_{1}^{*}, s_{2}^{*}, 0\right)$. At last, the direction of Hopf bifurcation and the stability of the bifurcating periodic orbits are discussed by applying the normal form theory and the center manifold theorem. A numerical example verifying our theoretical results is also correct.

\section{References}

[1] E. Beretta and Y. Takeuchi, Convergence results in SIR epidemic models with varying population sizes, Nonlinear Anal. 28 (1997), no. 12, 1909-1921.

[2] R. Bhattacharyya and B. Mukhopadhyay, Spatial dynamics of nonlinear prey-predator models with prey migration and predator switching, Ecological Complexity 3 (2006), 160-169.

[3] F. D. Chen and X. D. Xie, Permanence and extinction in nonlinear single and multiple species system with diffusion, Appl. Math. Comput. 177 (2006), no. 1, 410-426.

[4] J. A. Cui and L. S. Chen, The effect of diffusion on the time varying logistic population growth, Comput. Math. Appl. 36 (1998), no. 3, 1-9.

[5] S. J. Gao, L. S. Chen, and Z. D. Teng, Hopf bifurcation and global stability for a delayed predator-prey system with stage structure for predator, Appl. Math. Comput. 202 (2008), no. 2, 721-729.

[6] J. Hale, Theory of Functional Differential Equation, Springer-Verlag, 1977.

[7] J. Hale and S. Lunel, Introduction to Functional Differential Equations, Springer-Verlag, New York, 1993.

[8] B. Hassard, D. Kazarino, and Y. Wan, Theory and applications of Hopf bifurcation, Cambridge University Press, Cambridge, 1981.

[9] H. W. Hethcote, The mathematics of infectious diseases, SIAM Rev. 42 (2000), no. 4, $599-653$.

[10] Y. Kuang and Y. Takeuchi, Predator-prey dynamics in models of prey dispersal in twopatch environments, Math. Biosci. 120 (1994), no. 1, 77-98.

[11] G. H. Li and Z. Jin, Global stability of an SEI epidemic model, Chaos Solitons Fractals 21 (2004), no. 4, 925-931.

[12] G. H. Li and Z. Jin, Global stability of an SEI epidemic model with general contact rate, Chaos, Solitons and Fractals 23 (2005), no. 3, 997-1004.

[13] Z. Lu and Y. Takeuchi, Global asymptotic behavior in single-species discrete diffusion systems, J. Math. Biol. 32 (1993), no. 1, 67-77. 
[14] Prajneshu and P. Holgate, A prey-predator model with switching effect, J. Theoret. Biol. 125 (1987), no. 1, 61-66.

[15] S. G. Ruan and J. J. Wei, On the zeros of transcendental functions with applications to stability of delay differential equations with two delays, Dyn. Contin. Discrete Impuls. Syst. Ser. A Math. Anal. 10 (2003), no. 6, 863-874.

[16] B. Shulgin, L. Stone, and Z. Agur, Pulse vaccination strategy in the SIR epidemic model, Bull. Math. Bio. 60 (1998), 1-26.

[17] X. Y. Song and L. S. Chen, Persistence and global stability for nonautonomous predatorprey system with diffusion and time delay, Comput. Math. Appl. 35 (1998), no. 6, 33-40.

[18] C. J. Sun, Y. P. Lin, and M. A. Han, Stability and Hopf bifurcation for an epidemic disease model with delay, Chaos, Solitons and Fractals 30 (2006), no. 1, 204-216.

[19] Y. Takeuchi, J. A. Cui, R. Miyazaki, and Y. Satio, Permanence of dispersal population model with time delays, J. Comput. Appl. Math. 192 (2006), no. 2, 417-430.

[20] M. Tansky, Switching effect in prey-predator system, J. Theoret. Biol. 70 (1978), no. 3, 263-271.

[21] E. I. Teramoto, K. Kawasaki, and N. Shigesada, Switching effect of predation on competitive prey species, J. Theoret. Biol. 79 (1979), no. 3, 303-315.

[22] R. Xu and Z. E. Ma, Stability and Hopf bifurcation in a ratio-dependent predator-prey system with stage structure, Chaos, Solitons and Fractals 38 (2008), no. 3, 669-684.

[23] K. Yang, Delay Differential Equations With Applications in Population Dynamics, Academic Press, INC, 1993.

[24] S. L. Zhang and H. Q. Fang, The dispersal properties of a class of epidemic models, J. Biomath. 14 (1999), no. 3, 264-268.

[25] T. Zhao, Y. Kuang, and H. L. Simith, Global existence of periodic solutions in a class of delayed Gause-type predator-prey systems, Nonlinear Anal. 28 (1997), no. 8, 1373-1394.

[26] X. Y. Zhou, X. Y. Shi, and X. Y. Song, Analysis of nonautonomous predator-prey model with nonlinear diffusion and time delay, Appl. Math. Comput. 196 (2008), no. $1,129-136$.

Changuin Xu

Guizhou Key Laboratory of Economics System Simulation

Guizhou College of Finance and Economics

GuiYang 550004, P. R. China

E-mail address: xcj403@126.com

MAOXIN LiaO

School of Mathematical Science and Computing Technology

Central South University

Changsha, Hunan 410083, P. R. China

E-mail address: maoxinliao@163.com 